Nota añadida /Added note: 06-11-2017

Ver fe de erratas al final de este documento / See erratum at the end of this document

\title{
Estudio petrográfico, geoquímico y mineralógico de la fulgurita San José de Lourdes, Zacatecas, México
}

\section{Adela Margarita Reyes-Salas ${ }^{1, *}$, Consuelo Macías-Romo ${ }^{1}$, Fernando Ortega-Gutiérrez ${ }^{1}$, Eugenio Octavio Reyes-Salas ${ }^{2}$, Daniel Flores-Gutiérrez ${ }^{3}$, Leticia Alba-Aldave ${ }^{1}$, Patricia Girón-García ${ }^{1}$, Jasinto Robles-Camacho ${ }^{4}$, José Luis García-Martínez ${ }^{1}$ y Karina Elizabeth Cervantes-de la Cruz $^{5}$}

\author{
${ }^{1}$ Universidad Nacional Autónoma de México, Instituto de Geología, Del. Coyoacán, 04510, Ciudad de México, México. \\ ${ }^{2}$ Universidad Nacional Autónoma de México, Facultad de Química, Del. Coyoacán, 04510, Ciudad de México, México. \\ ${ }^{3}$ Universidad Nacional Autónoma de México, Instituto de Astronomía, Del. Coyoacán, 04510, Ciudad de México, México. \\ ${ }^{4}$ Instituto Nacional de Antropología e Historia, Subdirección de Laboratorios y Apoyo Académico, Centro INAH Michoacán, México. \\ ${ }^{5}$ Universidad Nacional Autónoma de México, Facultad de Ciencias, Del. Coyoacán, 04510, Ciudad de México, México. \\ *adelars@unam.mx
}

\section{RESUMEN}

En este trabajo se presentan los resultados del estudio petrográfico, geoquímico y mineralógico de fulguritas originadas en suelo calcáreo ubicadas al noroeste de Fresnillo en las inmediaciones de San José de Lourdes, en el Estado de Zacatecas, México. Las fulguritas, principalmente tienen formas tubulares, y están constituidas por vidrio vesicular de composición heterogénea comparable, químicamente, con andesitas. Ellas contienen en su matriz formas diversas de crecimiento incipiente de cristales de wollastonita y un silicato de Fe, no determinado; destacan también fragmentos de cuarzo formando esferoides rodeados de vidrio, -similares a los llamados cuarzo ballen (ballen quartz), característicos de las rocas de impacto-, cristobalita y granos escasos de ilmenita, apatita, circón, plata y oro. También incluyen algunas aleaciones micrométricas de $\mathrm{Si}$-Fe (siliciuros) en composiciones variables y compuestos de arsénico-calcio, estos últimos reportados por primera vez en fulguritas. Con base en la formación de microlitos dentro del vidrio y fusión del cuarzo, se estimaron temperaturas pico de cristalización superiores a $1,700^{\circ} \mathrm{C}$.

Palabras clave: fulgurita; wollastonita; cristobalita; aleaciones de $\mathrm{Si}-\mathrm{Fe}$; As-Ca; cuarzo ballen; Zacatecas; México.

\footnotetext{
ABSTRACT

Petrographic, chemical and mineralogical studies were performed on a fulgurite formed by melting of soil by lightning strike located at San José de Lourdes, northeast of Fresnillo, State of Zacatecas, Mexico. Polarizing microscopy, scanning electron microscopy with EDS, microprobe WDS analyses, micro-Raman spectroscopy, X-ray diffraction and $X$-ray fluorescence were used as analytical methods. Most of the specimens
}

are tubular and have a smooth, lustrous, black, glassy texture while the exterior is encrusted with soil grains. Internally, this fulgurite is partly composed of heterogeneous vesicular glass mostly of andesitic composition containing fragments of skeletal crystals of wollastonite and undetermined Fe- silicate, spheroids of $\mathrm{SiO}_{2}$ similar to ballen quartz within the glass. The presence of very fine and scarce grains of ilmenite, zircon, apatite, silver and gold besides some Si-Fe alloys (silicides) of variable composition and arsenic-calcium compounds (which is found for the first time in fulgurites) is also reported. We estimate that the temperatures of crystallization were above $1,700^{\circ} \mathrm{C}$ considering into account the melting of quartz and presence of reduced phases.

Key words: fulgurite; wollastonite; cristobalite; Si-Fe alloy; As-Ca; ballen quartz; Zacatecas; Mexico.

\section{INTRODUCCIÓN}

Las fulguritas, (del latín fulgur, relámpago) son el resultado de la fusión y reducción química con enfriamiento rápido de materiales de la superficie o cercanos a la superficie en la Tierra por el impacto de relámpagos (Heide y Heide, 2011). La mayoría de los relámpagos que inciden en la Tierra alcanzan temperaturas de $2,500{ }^{\circ} \mathrm{C}$ (Ege, 2005) y los tiempos de fusión inducidos por los relámpagos son del orden de un segundo (Grapes, 2006). Existen variedades de fulguritas llamadas seudofulguritas que se han formado por la combinación de la energía del relámpago y la contribución de una línea de energía de alto voltaje, como el caso de la fulgurita Farmington del Estado de Connecticut (Estados Unidos de América) (Sheffer et al., 2003, 2006); la fulgurita El Rosario del Estado de Hidalgo (México) (Ortiz-Hernández y Hernández-Pérez, 1996; Ramírez-Cardona et al., 2006) y la fulgurita Zacatecas (México) (Reyes-Salas et al., 2006).

Reyes-Salas, A.M., Macías-Romo, C., Ortega-Gutiérrez, F., Reyes-Salas, E.O., Flores-Gutiérrez, D., Alba-Aldave, L., Girón-García, P., Robles-Camacho, J., GarcíaMartínez, J.L., Cervantes-de la Cruz, K.E., 2017, Estudio petrográfico, geoquímico y mineralógico de la fulgurita San José de Lourdes, Zacatecas, México: Revista Mexicana de Ciencias Geológicas, v. 34, núm. 3, p. 170-181. 
En comparación con la gran cantidad de investigaciones realizadas en fulguritas formadas en arenas, las cuales se caracterizan por constituir formas tubulares con huecos centrales, aquellas formadas en rocas y suelos son muy escasas (Switzer y Melson, 1972); las fulguritas de origen rocoso por lo general forman costras o fragmentos no tubulares (p. ej., la fulgurita Monte Ararat, en Turquía; Goedeke y Techmer, 1999; Sheffer et al. 2006). También han sido reportadas fulguritas de serpentina con níquel y aún algunas raras compuestas de hierro y de óxido de manganeso (Riley, 1959). Entre las escasas investigaciones de fulguritas que se originan en suelos, figuran los trabajos de Hawkins (1960, suelo arcilloso de Ontario en Canadá) y de Gifford (1999, suelo arcilloso en Australia). Essene y Fisher (1986), encuentran y estudian una de las fulguritas más grandes conocida (Winans Lake) con aproximadamente $5 \mathrm{~m}$ de largo y casi $30 \mathrm{~cm}$ en diámetro en suelos de Ann Arbor, Michigan, (Estadis Unidos de América). Los estudios de Essene y Fisher (1986) mostraron que la fulgurita de Winans Lake contiene minerales no conocidos en forma natural en la Tierra, pues son reducidos químicamente o deoxidizados y forman los siliciuros. Los trabajos de la fulgurita mexicana formada en suelo arcilloso en la localidad El Rosario (Estado de Hidalgo), destaca por su contenido de grandes esferoides de siliciuros (Ortíz-Hernández y Hernández-Pérez, 1996; Ramírez et al., 2006).

Los estudios químicos y microestructurales de fulguritas resultan interesantes por su naturaleza, ya que al producirse la redistribución de materia instantáneamente, se llevan a cabo fenómenos poco comunes. Por lo tanto, estos fenómenos pueden considerarse entre aquellos aún más insólitos como los impactos meteoríticos, o en especial los que muestran los condros de las meteoritas condríticas. También deben considerarse otros procesos de fusión a temperatura alta presentes en cenizas volcánicas, impactitas, microesferas cósmicas, así como comparar con aquellos productos reducidos como los siliciuros que se encuentran tanto en fulguritas como en algunas meteoritas (Parnell et al., 2008). Los trabajos de Macdonald et al. (2004), Ende et al. (2012) y Carter et al. (2010) sugieren que los relámpagos generan alta temperatura y presiones momentáneas suficientemente elevadas como para producir cristales de cuarzo con crucero, coesita y stishovita (p. ej. la fulgurita Edeowie de Australia). Los cristales de cuarzo de impacto encontrados en fulguritas del Sahara y en la fulgurita de Greensboro (Carolina del Norte, Estados Unidos de América) indican un estado de deformación con presión de choque de $25 \mathrm{GPa}$ (Ende et al., 2012; Carter et al., 2010). El cuarzo y sus polimorfos son los minerales más comunes como indicadores de metamorfismo de impacto o de choque y permite la calibración de la presión. El cuarzo presenta una amplia variedad de deformaciones y transformaciones debidas a los mecanismos inducidos por choque, entre las que se encuentra la formación de cuarzo ballen (ballen quartz) con implicaciones termodinámicas significativas en el metamorfismo de choque (Ferriére et al., 2009; Schmieder y Buchner, 2007; Mokhtari, 2014; Kuriyama et al., 2011; Cartens, 1975). Sin embargo, las presiones involucradas en la formación de fulguritas aún no están bien determinadas, ya que en la generalidad de trabajos sobre fulguritas no se han observado las características de los efectos de alta presión.

Con el fin de contribuir al conocimiento de las características y condiciones de formación de las fulguritas se presenta el estudio petrográfico, geoquímico y mineralógico de las fulguritas de suelo encontradas en San José de Lourdes, cerca de Fresnillo, Estado de Zacatecas, México. Las técnicas utilizadas en este estudio incluyen láminas delgadas con microscopio petrográfico, microscopio electrónico de barrido con energía dispersiva de rayos X (EDS), microsonda electrónica (WDS), espectroscopía micro-Raman, y difracción y fluorescencia de rayos $\mathrm{X}$.

\section{CARACTERÍSTICAS GENERALES Y DIVERSIDAD DE LAS FULGURITAS}

Las fulguritas pueden ser divididas en tres clases de acuerdo al material de origen: fulguritas de arena, de suelo y de roca. Las fulguritas de arena formadas en playas o regiones desérticas son las más comunes, principalmente aquellas cuya composición es casi totalmente de $\mathrm{SiO}_{2},(90-99 \%$ en peso), recibiendo entonces el nombre de lechatelierita (vidrio puro de sílice). Posiblemente estas fulguritas son mucho más frecuentes, dada la facilidad que tienen los rayos de penetrar en materiales no consolidados (Lozano-Fernández et al., 2007). Las fulguritas de suelo son menos conocidas posiblemente por su susceptibilidad a la desintegración en el suelo donde se formaron. En cuanto a las fulguritas de roca, a pesar de ser más resistentes a la erosión, existen pocos reportes. El vidrio de estas dos últimas puede ser relativamente pobre en sílice y presentar una variedad amplia de colores, dependiendo de la composición del material madre.

Las fulguritas también presentan una gran variedad de formas, aunque las más abundantes son tubulares. Burbujas y vesículas son componentes comunes en toda fulgurita, indicando que al proceso de fusión ocurrió paralelamente el de ebullición y evaporación (Goedeke y Techmer, 1999). La cantidad, tamaño y forma de burbujas y vesículas son muy variables, y se atribuye a la expansión de los gases, tales como el aire y el vapor de agua generados en el proceso de impacto (Lozano-Fernández et al., 2007). La mayoría de las fulguritas son tubos irregulares y huecos. Los tubos están constituidos por vidrio en su parte interna y en su exterior es común que se describa como una superficie muy rugosa, esto es atribuido a fragmentos de minerales y rocas que no fueron totalmente fundidos y que son incorporados como parte de su estructura. Por lo general son delicadas y se fragmentan fácilmente. Cabe mencionar que la mayoría de las fulguritas tubulares se caracterizan por tener un centro hueco a través de casi toda su longitud; algunas pueden presentar paredes delgadas, lo cual las hace muy frágiles. Otras, como el caso de la fulgurita en estudio, los tubos forman barras constituidas de vidrio vesicular.

En cuanto al tamaño, también hay una gran diversidad, por lo general, las fulguritas tienen en promedio un diámetro interno en el rango de milímetros-centímetros; excepcionalmente pueden tener varios metros de longitud (Essene y Fisher, 1986; Wright, 1999, LozanoFernández, et al., 2007). La formación de una fulgurita, así como la cantidad de masa fulgurítica, depende de varios factores: intensidad de la descarga del rayo, de la humedad en la atmósfera y en el suelo, composición del material madre, entre otros; la forma cilíndrica es el resultado de la tensión superficial, mientras que el diámetro depende de la cantidad de expansión de aire y humedad a lo largo de la ruta de descarga del relámpago (Grapes, 2006).

\section{Los siliciuros y su formación}

Algunas fulguritas pueden contener, raramente, esferoides metálicos de aleaciones de Si-Fe llamados siliciuros, que son fases extremadamente reducidas, los cuales también han sido reportadas en meteoritas, suelos lunares, en muestras de polvo estelar y en rocas terrestres del límite núcleo-manto (Bird y Weathers, 1975). Son fases minerales tienen diferentes composiciones de $\mathrm{Fe}$ y de $\mathrm{Si}$, que forman compuestos como: $\mathrm{FeSi}$ (ferrisilita), $\mathrm{FeSi}_{2}$ (ferdisilicita) y $\mathrm{Fe}_{2} \mathrm{Si}$ (hapkeita), los cuales se generan en condiciones dominantemente reductoras por volatilización extrema y temperaturas muy altas $>1800{ }^{\circ} \mathrm{C}$ (Essene y Fisher, 1986, Wasserman et al., 2002, Wasserman y Melosh, 2001, Jones et al., 2005, Sheffer et al., 2003). Estos siliciuros son sumamente interesantes de investigar, ya que son compuestos metaestables poco comunes. Las fulguritas permiten acceder al estudio de estas fases.

Uno de los mecanismos para la formación de siliciuros más 
sugerido por diversos autores es que son el resultado de procesos de vaporización-depósito. Essene y Fisher (1986), Wasserman et al., (2002) y Gopon et al., (2014), entre otros, sugieren como otro mecanismo que contribuye a la reducción extrema el material carbonoso de los suelos y la liberación de oxígeno durante la ebullición suministrados por el flujo de electrones a través del fundido, como ocurre en la electrólisis para formar óxido nítrico a partir del nitrógeno en la atmósfera ambiental. Así explican que, cuando en el sistema hay $\mathrm{SiO}_{2}$, antes del impacto, el material en estado sólido después del impacto es un líquido donde coexisten $\mathrm{SiO}_{2}$ y Si metálico en equilibrio con $\mathrm{SiO}_{\text {y }} \mathrm{O}_{2}$ en forma de gases y donde puede incorporarse Fe en el sistema en estados de oxidación con diferentes proporciones.

\section{LOCALIZACIÓN}

Las fulguritas objeto de este estudio se extienden en ramificaciones que ocupan un área semicircular de unos $4 \mathrm{~m}$ de diámetro y aproximadamente $2 \mathrm{~m}$ de profundidad. Están situadas dentro de un campo de cultivo ubicado al noroeste de Fresnillo en las inmediaciones de San José de Lourdes, Estado de Zacatecas, México (Figura 1), cuyo suelo es clasificado como Xerosol Cálcico, donde la vegetación que predomina es mezquital, matorral desértico y pastizal natural (INEGI, 2007).

A un lado del área se encontraron restos de un poste de cableado eléctrico, por lo que podría suponerse que el rayo pudo impactar al poste y la energía eléctrica de los cables contribuir al evento, por lo que podría considerarse una seudofulgurita. El área ha sido excavada por los habitantes del lugar, quienes han considerado estos materiales como fragmentos de meteoritas. Se ignora la fecha exacta del evento que formó estas fulguritas, pero posiblemente fue en los inicios de la década de 1990.

\section{DESCRIPCIÓN MEGASCÓPICA}

La morfología de estas fulguritas es diversa, y las estructuras tubulares vítreas, de tamaños variables son predominantes ( $1 \mathrm{~mm}-30 \mathrm{~cm}$ de longitud y de $0.1 \mathrm{~mm}-10 \mathrm{~mm}$ de diámetro). En su mayoría, el color interior es negro con lustre vítreo, con gran cantidad de vesículas esferoidales con diámetro de 0.1-10 $\mathrm{mm}$. La superficie exterior (costra o corteza) de la fulgurita de forma tubular, es de aspecto terroso, de grosor milimétrico y cubierta de granos parcialmente fusionados y de color blanco amarillento, similar al suelo del campo de cultivo. Los tubos forman barras de vidrio que además de vesículas presentan algunas burbujas (Figura 2a, 2b).

También se encontraron fulguritas en forma de costras con lustre vítreo y con gran cantidad de vesículas (Figura 2c) que, igualmente, forman fragmentos masivos con vesículas y sin lustre de hasta $15 \mathrm{~cm}$ de diámetro (Figura 2d). Algunos fragmentos con aproximadamente $7 \mathrm{~cm}$ de diámetro de costras vítreas presentan colores verdosos y rojizos en su superficie.

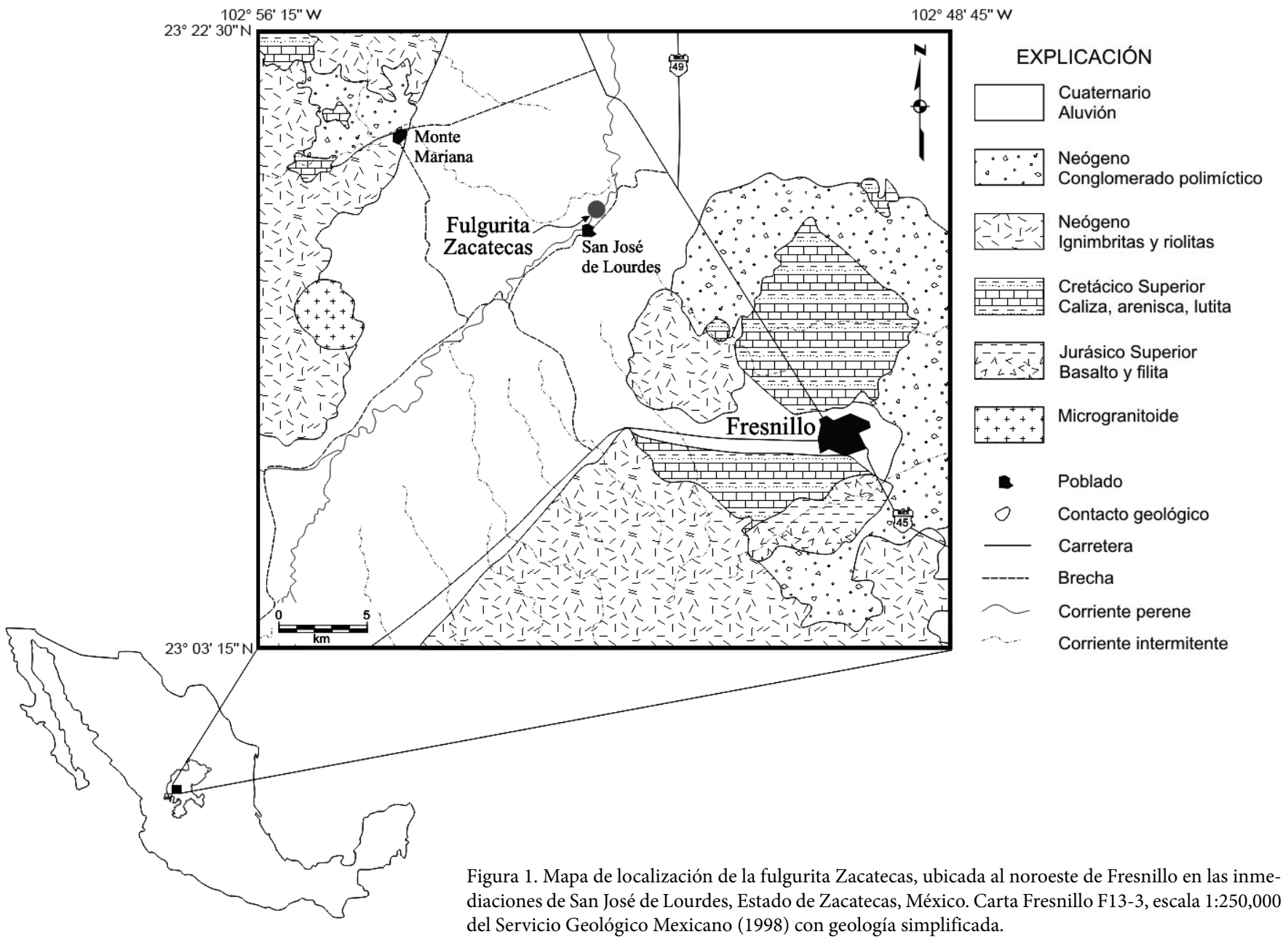


En el material tubular es posible observar cúmulos de material blanco de tamaño milimétrico dispersos en la matriz vítrea y en las cercanías de las vesículas. (Figura 2a).

\section{MÉTODOS Y TÉCNICAS DE ESTUDIO}

El material colectado fue estudiado por diversas técnicas analíticas: microscopio petrográfico, microscopía electrónica de barrido y microsonda electrónica, técnicas de rayos $\mathrm{X}$, tanto difracción como fluorescencia y espectroscopía Raman.

\section{Microscopio petrográfico}

Los estudios petrográficos involucraron la preparación de secciones delgadas pulidas en diferentes direcciones de las fulguritas tubulares con sus costras. Los vidrios fueron inicialmente estudiados utilizando un microscopio de luz polarizada marca Leitz, modelo "Loborlux 12 pol”, con el que se fotografiaron y seleccionaron las zonas de interés para los estudios analíticos posteriores.

\section{Microscopio electrónico de barrido y Microsonda electrónica}

Posteriormente, secciones delgadas y pequeños fragmentos fue-
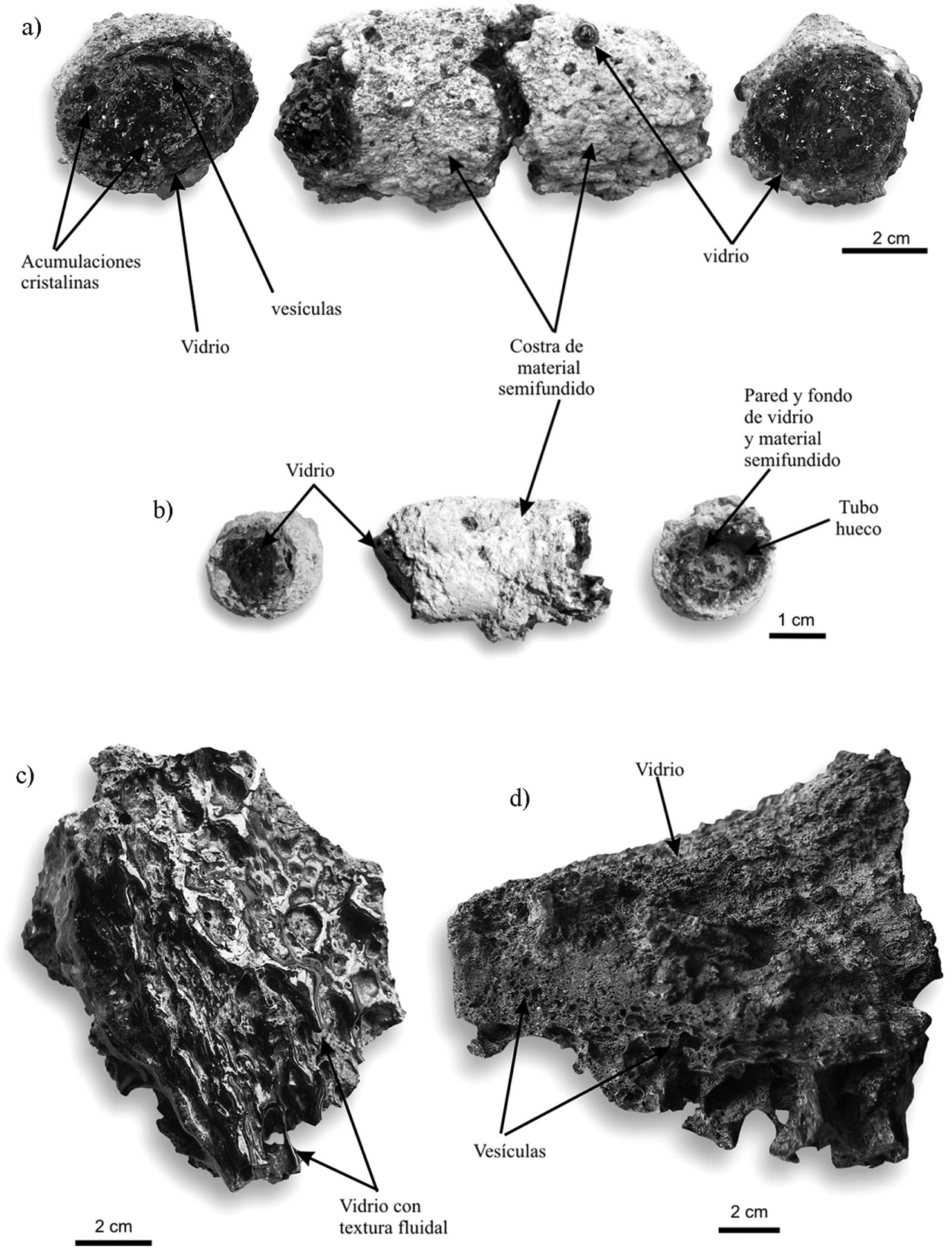

Figura 2. Fulgurita Zacatecas. a) y b) Dos aspectos de las formas tubulares de la fulgurita. Al centro, imagen longitudinal con vistas transversales de los extremos de los tubos. Se distingue en su interior el material vítreo de color negro conteniendo vesículas y pequeñas acumulaciones cristalinas de cuarzo, cristobalita o wollastonita; en su exterior, la costra, constituída de material adherido semifundido de color claro de aspecto terroso. c) Fragmento de pared del ramal principal de donde se extienden los tubos de vidrio; la pared está formada por vidrio opaco muy vesicular y sobre ésta, el material vítreo con textura fluidal de color negro. d) Fragmento vítreo opaco, muy vesicular, en la pared del ramal principal de la fulgurita. 
ron colocados sobre cinta adhesiva y recubiertos por carbón con una evaporadora JEOL JEE-4X. Las secciones delgadas fueron analizadas por espectrometría WDS (energía dispersiva de longitud de onda de rayos X) con una microsonda electrónica de barrido JEOL JXA-8900R en el Laboratorio Universitario de Petrología de la UNAM. Se utilizaron estándares de silicatos. Se efectuaron análisis puntuales en áreas diversas y análisis puntuales lineales a intervalos de $50 \mu \mathrm{m}$ a distancias de $1000 \mu \mathrm{m}$; se utilizaron $15 \mathrm{kV}, 5 \mu \mathrm{m}$ de diámetro del haz y $10 \mathrm{nA}$ de corriente para el vidrio, reduciendo el diámetro del haz a $1 \mu \mathrm{m}$ para los microlitos. En el caso de las aleaciones de Si-Fe y As-Ca, solamente se efectuaron análisis de EDS debido a su tamaño micrométrico y porque, en su mayoría, fueron detectados sobre superficies sin pulir. Con un microscopio electrónico de barrido JEOL 6300 se obtuvieron imágenes con electrones retrodispersados, tanto en secciones delgadas como en los fragmentos.

\section{Estudios por fluorescencia y difracción de rayos $\mathrm{X}$}

Muestras del material encajonante (madre), de la corteza y del vidrio de la fulgurita Zacatecas fueron preparadas para ser analizadas por el método de fluorescencia de rayos X (LFRX -SIEMENS SRS 3000) para determinar la composición química de elementos mayores y elementos traza. Los análisis fueron efectuados en el Laboratorio Universitario de Geoquímica Isotópica (LUGIS) de la UNAM de acuerdo a la metodología propuesta por Bernal y Lozano-Santacruz (2005).

La difracción de rayos X (DRX) se aplicó a un fragmento de la fulgurita que se pulverizó en mortero de ágata hasta obtener partículas de dimensiones entre 50 y 30 micómetros. Se utilizó un difractómetro Siemens modelo D-5000, Philips modelo 1130/96 (generador) y PW 1050/25 (goniómetro), con radiación CuKa, en el intervalo angular $2 \theta$ de 4 a $70^{\circ}$ a fin de conocer fases mineralógicas de nueva formación contenidas en el vidrio de la fulgurita.

\section{Espectroscopía Raman}

El estudio con espectroscopía Raman se realizó en dos secciones delgadas al igual que en pequeños fragmentos de tubos y costra. Se utilizó un espectrómetro dispersivo Almega XR equipado con un microscopio Olympus (BX51), con un diámetro de haz de $\sim 1.5 \mu \mathrm{m}$, tanto para enfocar el láser en la muestra como para colectar la luz dispersada en una configuración retrodispersiva de $180^{\circ}$. El espectro Raman se acumuló por $25 \mathrm{~s}$ con una resolución de $\sim 4 \mathrm{~cm}^{-1}$. La fuente de excitación fue una radiación de $532 \mathrm{~nm}$ de un láser de $\mathrm{Nd}: \mathrm{YVO}_{4}$ (frecuencydoubled) y la potencia incidente en la muestra fue de $\sim 10 \mathrm{~mW}$.

Se colocan las láminas delgadas en la platina del microscopio, se enfoca el mineral que se quiere identificar y se cambia la función de microscopio óptico a microscopio Raman, haciendo pasar el rayo láser por el objetivo. La luz dispersada que regresa por el mismo objetivo se hace pasar por un filtro Noch para eliminar la luz de longitud de onda de $532 \mathrm{~nm}$; el resto del haz luminoso incide en una rejilla que separa las diferentes longitudes de onda y finalmente llega al detector CCD. El resultado es un espectro en el cual cada banda representa una forma de vibrar de los átomos del cristal, que a su vez representan la estructura del cristal.

\section{RESULTADOS}

\section{Petrografía, composición química y mineralogía}

\section{Microscopía óptica}

Los vidrios de la fulgurita Zacatecas son incoloros, ópticamente isotrópicos, presentan texturas de flujo y esferoides de cuarzo (Figura 3a). En ciertas áreas, se encontró cúmulos de material blanquecino cons- tituidos por pequeños cristales principalmente de wollastonita, (Figura 3b-3d). También se observó una gran cantidad de vesículas que indican vaporización de los materiales fundidos y alrededor de algunas de ellas se acumulan las formaciones cristalinas de wollastonita (Figura 3b-3d).

\section{Microscopía electrónica}

El vidrio es vesicular y contiene los esferoides de cuarzo similar al cuarzo ballen (ballen quartz), así como cristales incipientes cerca de las vesículas (Figuras $3 \mathrm{e}-3 \mathrm{~g}$ ). Los cristales de cuarzo ballen que, tienen tamaños entre 50 y $700 \mu \mathrm{m}$, están constituidos por granos esferoidales de $\mathrm{SiO}_{2}$ y localmente contienen fragmentos pequeños de Ag y Au de tamaño micrométrico (Figura 3g). En las Figuras 3e y $3 \mathrm{f}$, puede observarse la estructura típica de flujo del fundido y las diferentes tonalidades de grises en las imágenes obtenidas con electrones retrodispersados; los análisis elementales realizados con la microsonda electrónica en una sección $500 \mu \mathrm{m}$ (transecto $\mathrm{a}-\mathrm{b}$ ) en la matriz de la fulgurita muestran la heterogeneidad del vidrio a esta escala micrométrica (Figura 3e, Figura 4 y Tabla 1); los tonos más claros corresponden a los valores más altos de óxidos de hierro y de aluminio, los más oscuros contienen mayor porcentaje de óxido de calcio. En general, los análisis con la microsonda electrónica revelan un vidrio silicatado rico en $\mathrm{Ca}$. Los vidrios con contenidos variables de $\mathrm{SiO}_{2}$ entre $49.90-62.38 \%$ en peso, son particularmente ricos en calcio, entre $13.69-29.7 \%$ en peso (Tabla 1). Los vidrios tienen una composición química similar a la de las andesitas. Por considerar a la fulgurita como el resultado de material fundido, análogo al magmático, se realizó el cálculo de la Norma CIPW, obteniendo la formación de wollastonita normativa (Tabla 1). En la Figura 4, se muestra el contenido relativo de elementos mayores más representativos en el vidrio, los óxidos $\mathrm{SiO}_{2}-\mathrm{Al}_{2} \mathrm{O}_{3}-\mathrm{CaO}$, analizado con la microsonda electrónica, indicado con el transecto a-b mostrado en la Figura 3: se aprecia la heterogeneidad en su composición a este nivel microscópico (variación en mediciones en transecto de 500 micrómetros).

Sobre las paredes de las vesículas se nuclearon cristalitos incluidos en la matriz vítrea de tipo dendrítico (Figuras 3a, 3d, 3f). En estos cristales incipientes se hicieron varios análisis por elementos con la microsonda electrónica, permitiendo calcular la fórmula: $\left(\mathrm{En}_{1.24} \mathrm{Fs}_{1.56} \mathrm{Wo}_{97.2}\right)$ a partir del promedio de los análisis, la cual sugiere una composición tipo wollastonita. Estos cristalitos despliegan una gran variedad de formas esqueléticas: curvadas, aciculares, arborescentes y plumosas (Figuras 3a-3d, 3f).

Otra formación cristalina observada dentro de la matriz vítrea consiste de estructuras radiales-petaliformes, con un centro granuloso del que parten estructuras esqueléticas plumosas ("pétalos") (Figuras $5 \mathrm{a}, 5 \mathrm{c})$. Se realizaron algunas determinaciones con microsonda (WDS) en áreas donde se concentran estas formaciones. La composición corresponde a un silicato rico en $\mathrm{Fe}$, cuya naturaleza mineral no se ha determinado; también se observaron estas estructuras en fragmentos sin pulir (Figuras 5a, 5c; Tabla 2).

Las aleaciones metálicas de $\mathrm{Si}$-Fe (siliciuros) pueden observarse de forma prismática, en tamaños de alrededor de $10 \mu \mathrm{m}$ entre las formaciones del silicato rico en hierro (Figura 5a); también se encuentran dentro de la matriz vítrea y sobre las paredes de las burbujas (Figura 5 b y Tabla 3); así mismo, ocurren sobre algunas superficies vítreas donde son abundantes y se presentan alineadas (Figura 5b). Entre los siliciuros prismáticos alineados se observaron algunos cristalitos curvos con grandes cantidades de arsénico y calcio (Figura 5d, Tabla 4). Otras aleaciones ( $\mathrm{Si}-\mathrm{Fe}-\mathrm{Al}$ ) las encontramos con morfología de granular a prismática como precipitados sobre la superficie del vidrio (Figura 5e). Debido a que los siliciuros se observaron sobre una superficie sin pulir, sólo pudieron hacerse determinaciones semicuantitativas con EDS (Tabla 5). 

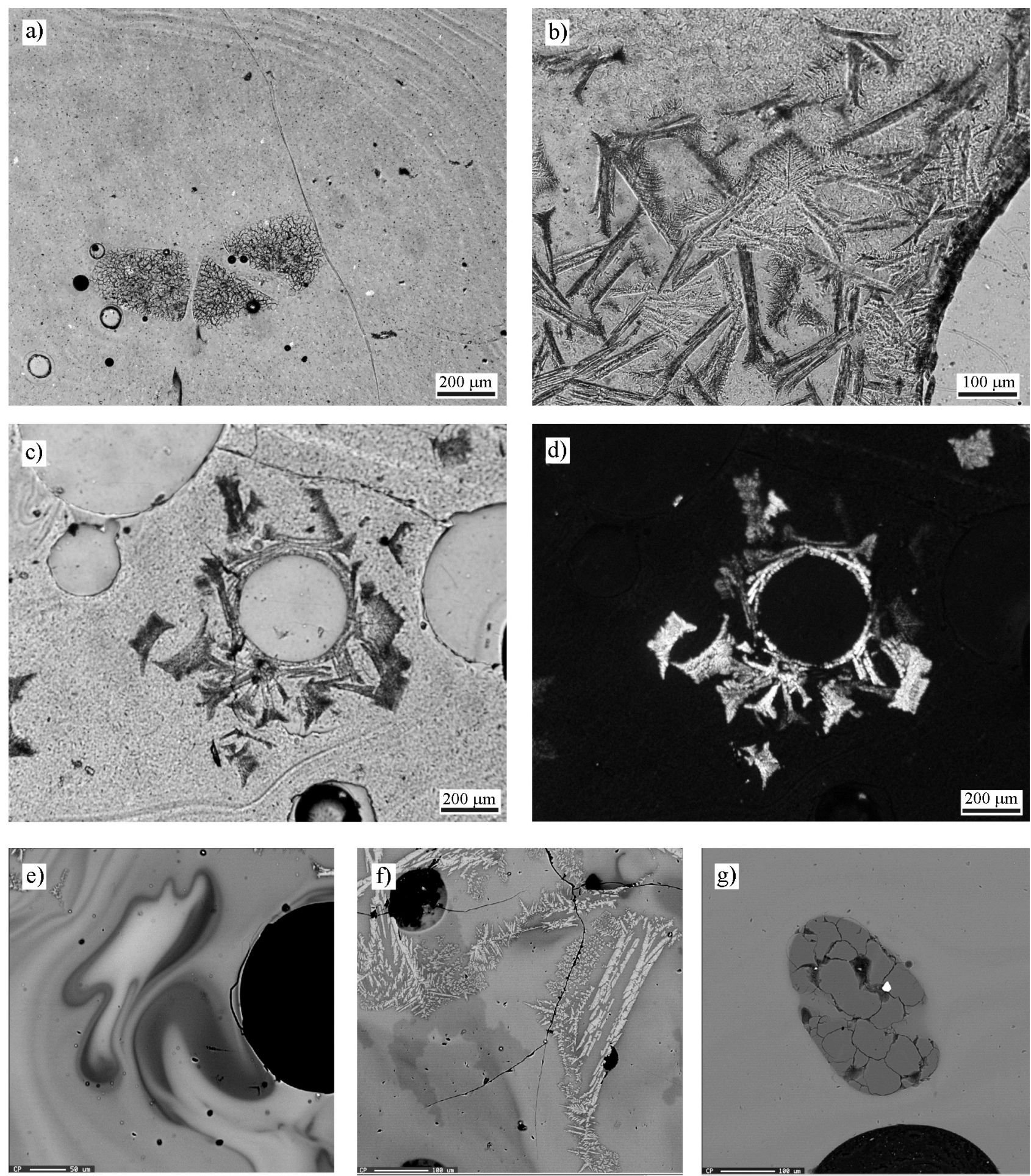

Figura 3. Fotomicrografías tomadas con microscopio petrográfico: a-c) Con luz natural; d) con luz polarizada. a) Parte del vidrio que conforma a la fulgurita Zacatecas con textura fluidal, pequeñas vesículas y hacia el centro, un esferoide alargado y separado en tres porciones formado por granos de $\mathrm{SiO}_{2}$ b) $\mathrm{Cristalitos}$ aciculares de wollastonita inmersos en vidrio, creciendo alrededor de una vesícula; al centro, textura plumosa. c) y d) Morfologías esqueléticas de wollastonita alrededor de una vesícula. e-g) Imágenes tomadas con electrones retrodispersados en el microscopio electrónico. e) Muestra, en tonos diferentes, la composición heterogénea del vidrio; los tonos más claros corresponden a los puntos analizados con mayor cantidad de aluminio y hierro. f) Cristales incipientes de wollastonita. g) Grano de cuarzo ballen el cual contiene dos partículas de oro y plata nativa; a la derecha, la partícula más brillante es oro. 


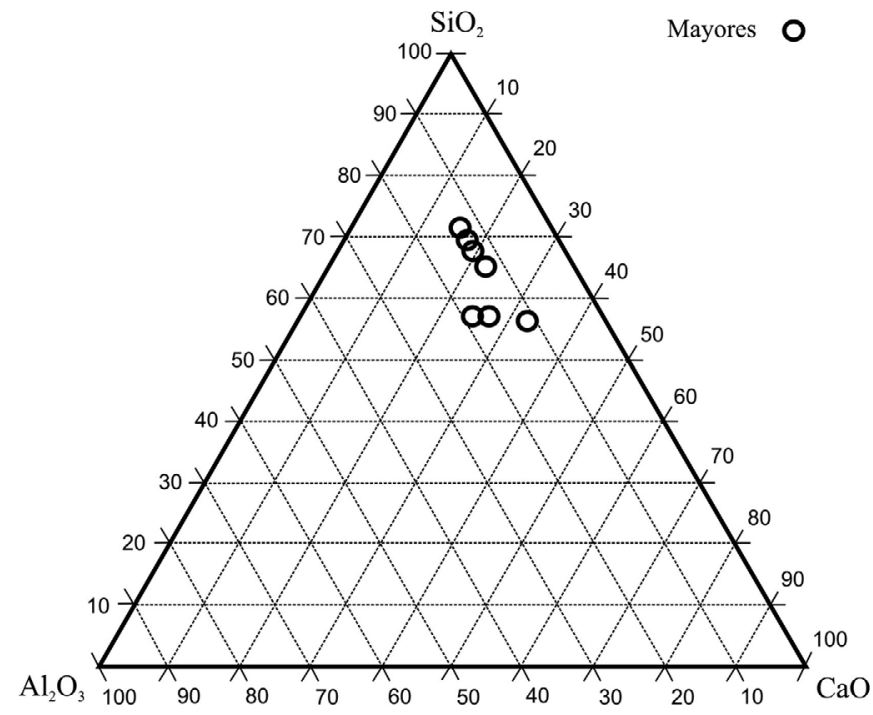

Figura 4. Resultados de los análisis con microsonda electrónica del vidrio de la fulgurita Zacatecas. Los círculos muestran el contenido relativo de $\mathrm{SiO}_{2}-\mathrm{Al}_{2} \mathrm{O}_{3}$ $\mathrm{CaO}$, observándose la heterogeneidad del vidrio.

Los óxidos y otros minerales que se presentan escasamente en la matriz vítrea corresponden a ilmenita, circón y apatita.

Rayos $X$

Mediante la técnica de DRX se identificó, además de la fase amorfa correspondiente al vidrio, calcita $\left[\mathrm{CaCO}_{3}\right]$, cuarzo $\left[\mathrm{SiO}_{2}\right]$, feldespatos, probablemente microclina intermedia $\left[\mathrm{KAlSi}_{3} \mathrm{O}_{8}\right]$ y fases de cristobalita (Figura 6).

La técnica de FRX permitió comparar la composición química del vidrio con la composición del suelo que originó a esta fulgurita y la corteza (cubierta de granos parcialmente fusionados), revelando volatización de elementos mayores durante la fusión. En la Tabla 6 se presentan los resultados de los análisis del suelo encajonante (madre), corteza y vidrio de la fulgurita Zacatecas. Se muestra pérdida notoria de $\mathrm{H}_{2} \mathrm{O}$ (\% en peso); también difieren de la composición del suelo porque se enriquece en $\mathrm{Si}_{2} \mathrm{O}, \mathrm{CaO}$ y $\mathrm{Al}_{2} \mathrm{O}_{3}$; la corteza presenta mayor cantidad de $\mathrm{Si}_{2} \mathrm{O}$ (71.83\%) posiblemente por contener mayor cantidad de granos de cuarzo semifundidos. En la Figura 7 puede observarse los principales elementos y su variación. En cuanto a los datos de elementos traza es notorio en el vidrio el enriquecimiento de $\mathrm{Zr}, \mathrm{Ba}, \mathrm{Rb}$, $\mathrm{Zn}, \mathrm{V}$ e Y con respecto al vidrio encajonante; en cambio, se empobrece notoriamente en Sr: la corteza presenta valores más similares a los del vidrio encajonante.

\section{Espectroscopía Raman}

De acuerdo a los espectros Raman obtenidos y comparándolos con los espectros publicados, se identificaron dos minerales: wollastonita y cuarzo (Figuras 8 y 9). El pico máximo característico que identifica al cuarzo es a $465 \mathrm{~cm}^{-1}$; en la gráfica obtenida para la fulgurita Zacatecas, este pico se muestra desplazado hacia la izquierda en $463 \mathrm{~cm}^{-1}$.

Se analizaron también otros minerales pero su espectro Raman en algunos casos mostraba mucha fluorescencia debido a las impurezas que contenían y en otros casos a que eran amorfos y no cristalinos.

\section{DISCUSIÓN}

La fusión y reducción de material silicatado por los impactos de relámpagos permiten entender y cuantificar los cambios producidos por efectos de temperaturas y probablemente presiones muy altas. Las fulguritas se forman en una gran variedad de tipos de suelos y rocas, son relativamente abundantes, y al encontrarse in situ permiten compararlas con el material adyacente. A diferencia de otros vidrios de impacto como las tectitas o los vidrios de cráteres de impacto que han sufrido erosión, por su abundancia y facilidad de obtención, las fulguritas resultan ser muestras ideales para investigar los procesos naturales de alta temperatura. La fulgurita de Zacatecas se formó en condiciones muy reductoras con volatilización extrema y temperaturas muy altas. Una comparación con su material materno indica una cantidad mínima de $\mathrm{H}_{2} \mathrm{O}$, como era de esperarse (Tabla 6). La

Tabla 1. Análisis con microsonda electrónica de óxidos mayores y norma CIPW que muestran la heterogeneidad de los vidrios que componen la fulgurita Zacatecas. Proporción de óxido en \% en peso.

\begin{tabular}{|c|c|c|c|c|c|c|c|}
\hline No. & 21 & 40 & 20 & 28 & 24 & 38 & 32 \\
\hline $\mathrm{SiO}_{2}$ & 49.70 & 49.90 & 50.96 & 57.46 & 59.47 & 60.65 & 62.38 \\
\hline $\mathrm{TiO}_{2}$ & 0.38 & 0.48 & 0.32 & 0.30 & 0.32 & 0.32 & 0.34 \\
\hline $\mathrm{Al}_{2} \mathrm{O}_{3}$ & 14.37 & 16.20 & 10.17 & 11.28 & 11.58 & 11.67 & 11.37 \\
\hline $\mathrm{FeO}$ & 3.43 & 4.60 & 3.62 & 3.36 & 3.61 & 3.15 & 3.06 \\
\hline $\mathrm{MgO}$ & 1.37 & 1.98 & 1.44 & 1.36 & 1.40 & 1.36 & 1.22 \\
\hline $\mathrm{MnO}$ & 0.04 & 0.06 & 0.03 & 0.05 & 0.01 & 0.05 & 0.00 \\
\hline $\mathrm{CaO}$ & 23.41 & 21.81 & 29.76 & 19.48 & 16.92 & 15.52 & 13.69 \\
\hline $\mathrm{Na}_{2} \mathrm{O}$ & 0.90 & 0.89 & 0.63 & 0.87 & 0.99 & 0.96 & 0.95 \\
\hline $\mathrm{K}_{2} \mathrm{O}$ & 4.29 & 4.19 & 2.37 & 3.46 & 3.51 & 3.87 & 4.35 \\
\hline Total & 97.88 & 100.10 & 99.29 & 97.62 & 97.82 & 97.54 & 97.35 \\
\hline Q & 0 & 0 & 0 & 12 & 16 & 18 & 21 \\
\hline or & 21 & 18 & 14 & 21 & 21 & 23 & 26 \\
\hline $\mathrm{ab}$ & 0 & 0 & 4 & 8 & 9 & 8 & 8 \\
\hline an & 23 & 28 & 18 & 17 & 17 & 16 & 14 \\
\hline di & 10 & 16 & 11 & 10 & 11 & 9 & 8 \\
\hline wo & 35 & 25 & 49 & 29 & 23 & 21 & 19 \\
\hline $\mathrm{mt}$ & 3 & 3 & 3 & 3 & 3 & 3 & 3 \\
\hline il & 1 & 1 & 1 & 1 & 1 & 1 & 1 \\
\hline
\end{tabular}



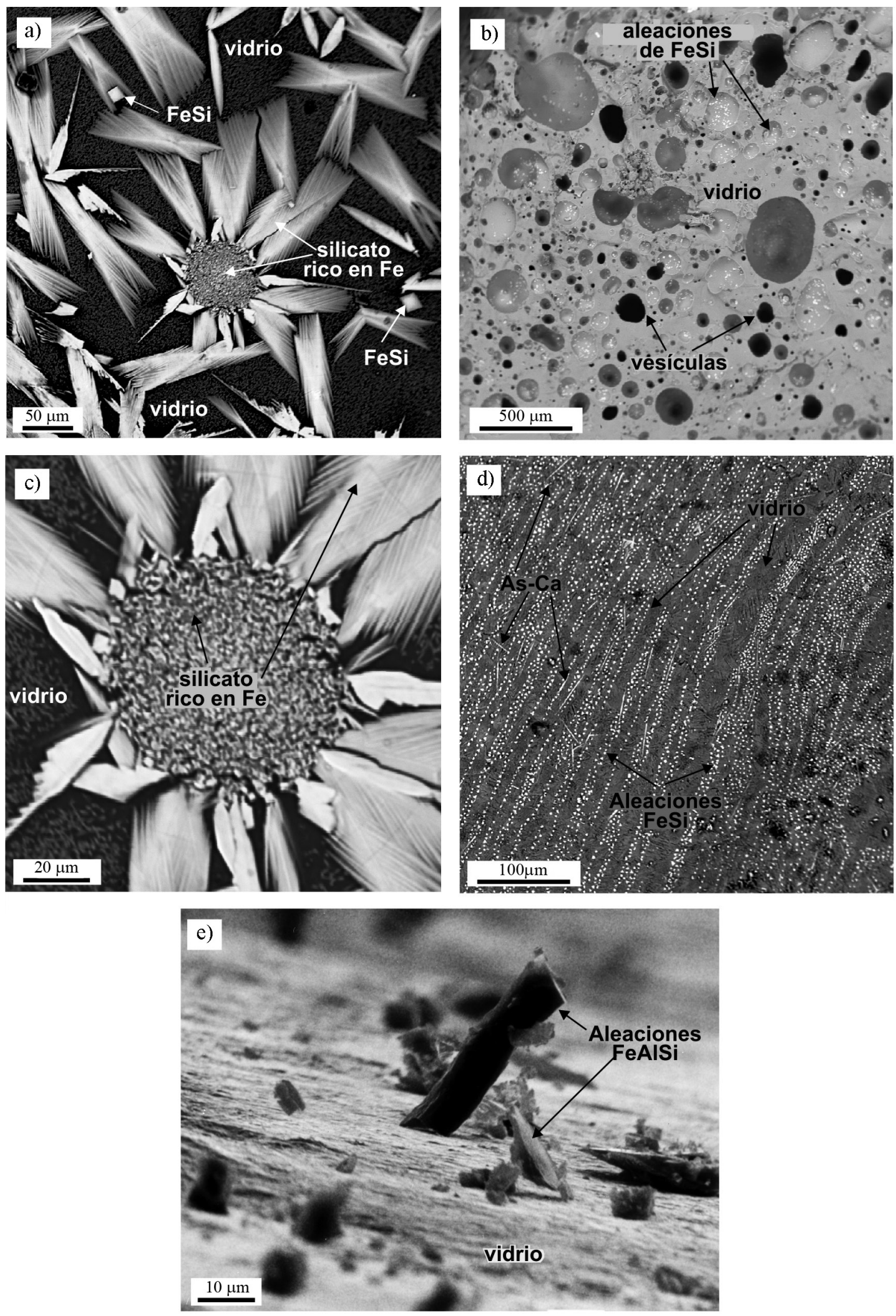

Figura 5. a) Formaciones cristalinas de silicato rico en Fe; en el centro presentan forma granular y los "pétalos" son formaciones esqueléticas de la misma composición. Los cristalitos prismáticos que se observan a la derecha arriba y a la izquierda abajo son aleaciones de Fe-Si (siliciuros); b) imagen de un fragmento pequeño sin pulir de la fulgurita Zacatecas, tomada con electrones retrodispersados. Se observa la gran cantidad de vesículas de diversos tamaños; los puntos blancos brillantes corresponden a las aleaciones de Si-Fe; c) detalle de la imagen a); d) micro-aleaciones alineadas de Fe-Si encontradas en la superficie rojiza de un fragmento de la fulgurita Zacatecas; las agujas, escasas en comparación con los siliciuros alineados, son compuestos de Ca ricos en As; e) aleaciones de Fe-Si-Al depositadas en la superficie del vidrio. 
Tabla 2. Análisis seleccionados de la fulgurita Zacatecas obtenidos por microsonda electrónica (WDS) de formaciones esqueletales (silicato rico en Fe).

\begin{tabular}{lrrr}
\hline \multicolumn{3}{c}{ Proporción de óxidos (\% en peso) } \\
\hline $\mathrm{SiO}_{2}$ & 23.45 & 26.16 & 29.93 \\
$\mathrm{TiO}_{2}$ & 0.01 & 0.01 & 0.02 \\
$\mathrm{Al}_{2} \mathrm{O}_{3}$ & 6.60 & 3.36 & 8.09 \\
$\mathrm{FeO}$ & 63.65 & 63.66 & 56.00 \\
$\mathrm{MnO}$ & 0.14 & 0.29 & 0.15 \\
$\mathrm{MgO}$ & 0.40 & 1.12 & 0.62 \\
$\mathrm{CaO}$ & 2.23 & 2.91 & 2.89 \\
$\mathrm{Na}{ }_{2} \mathrm{O}$ & 0.11 & 0.63 & 0.20 \\
$\mathrm{~K}_{2} \mathrm{O}$ & 2.49 & 1.45 & 2.78 \\
$\mathrm{Cr}_{2} \mathrm{O}_{3}$ & 0.11 & 0.00 & 0.22 \\
$\mathrm{Total}$ & 99.17 & 99.59 & 100.89 \\
\hline
\end{tabular}

wollastonita y el silicato rico en $\mathrm{Fe}$, que presentan morfologías típicas de congelamiento (Figuras $3 \mathrm{~b}-3 \mathrm{~d}$, $3 \mathrm{f}$ y Figuras $5 \mathrm{a}, 5 \mathrm{c}$ ), sugieren una cristalización rápida de un fundido en respuesta a una tasa elevada de enfriamiento y densidades bajas de nucleación (Essene y Fisher, 1986; Donaldson, 1976; Osinski, 2003). Esto es muy similar a lo que ocurre con basaltos submarinos (Bryan, 1972), así como en vidrios de impacto "congelados" rápidamente (Hörz et al., 2002) y en rocas lunares (Lofgren, 1971). Las formaciones esqueléticas de wollastonita son evidencia de enfriamiento rápido del material fundido por el impacto del relámpago; algo similar ocurre, por ejemplo, con fases esqueléticas de wustita $(\mathrm{FeO})$ de la fulgurita de Nigoziro, estudiada por Lysiuk y Lisiuk (2005). Por lo común, la composición química de la fulgurita puede ser comparada con la roca encajonante; en este caso, la gran cantidad de calcita y cuarzo en el suelo protolítico posiblemente propició la formación de wollastonita como mineral pirometamórfico.

La matriz del vidrio muestra estructuras de vidrio turbulento, indicando que el evento debió ser tan rápido que el vidrio no se homogeneizó a pesar de su fluidez. En la fulgurita Zacatecas también se observa, como inclusiones dentro del vidrio, cuerpos alargados (ovoides) en tamaños de 50 a $700 \mu \mathrm{m}$ constituidos por aglomeración de partículas esferoidales de 20 a $50 \mu \mathrm{m}$ (Figura 3g), compuestas de $\mathrm{SiO}_{2}$; estas son estructuras similares a los llamados cuarzo ballen (ballen quartz), que han sido reportados en varios cráteres de impacto terrestres (Ferriére et al., 2009). La formación de cuarzo ballen involucra un impacto con transición de sólido-sólido, desde un cristal de cuarzo a vidrio (lechatelierita), seguido por la formación de ballen de cristobalita beta y/o cuarzo beta $y$, finalmente, transformación a cristobalita alfa y/o cuarzo alfa, indicando temperaturas por arriba de $\operatorname{los} 1,700^{\circ} \mathrm{C}$, ya que varios autores han calculado la temperatura de fusión del cuarzo entre 1,700 y $2,000{ }^{\circ} \mathrm{C}$ (Grapes, 2006). Los cristales de cuarzo de impacto que han sido encontrados en algunas fulguritas pueden asignarse a un estado de deformación con presión de choque provocado por la acción del relámpago de alrededor de $25 \mathrm{GPa}$ (Ende et al., 2012). En la fulgurita Zacatecas ópticamente no se encontró cuarzo de cho-

Tabla 3. Análisis seleccionados de aleaciones granulares de Fe-Si de la Fulgurita Zacatecas obtenidos por energía dispersiva de rayos X (EDS). Normalizados al $100 \%$.

\begin{tabular}{lccccc}
\hline \multicolumn{5}{c}{ Proporción de elementos (\% en peso) } \\
\hline $\mathrm{Si}$ & 9.57 & 14.24 & 15.07 & 19.45 & 52.76 \\
$\mathrm{Fe}$ & 91.43 & 85.76 & 84.93 & 80.45 & 47.24 \\
Total & 100 & 100 & 100 & 100 & 100 \\
\hline
\end{tabular}

Tabla 4. Análisis seleccionados de minerales de As-Ca de la fulgurita Zacatecas, obtenidos por energía dispersiva de rayos X (EDS). Normalizados al $100 \%$.

\begin{tabular}{lrrr}
\hline & \multicolumn{3}{c}{ Proporción de elementos (\% en peso). } \\
\hline $\mathrm{Si}$ & 2.52 & 3.50 & 5.29 \\
$\mathrm{Ti}$ & 0.00 & 2.00 & 0.19 \\
$\mathrm{Al}$ & 0.60 & 1.40 & 1.35 \\
$\mathrm{Fe}$ & 10.30 & 7.50 & 7.51 \\
$\mathrm{Mn}$ & 2.70 & 2.70 & 2.70 \\
$\mathrm{Mg}$ & 12.80 & 12.77 & 12.70 \\
$\mathrm{Ca}$ & 25.00 & 20.60 & 20.63 \\
$\mathrm{Na}$ & 1.00 & 2.60 & 2.63 \\
$\mathrm{~K}$ & 0.60 & 1.30 & 1.29 \\
$\mathrm{As}$ & 43.70 & 45.00 & 44.67 \\
$\mathrm{~S}$ & 0.60 & 0.95 & 0.95 \\
Total & 100.00 & 100.00 & 100.00 \\
\hline
\end{tabular}

que, sin embargo el pico del espectro Raman (Figura 9) obtenido a $463 \mathrm{~cm}^{-1}$, se encuentra desplazado con respecto a los cristales de cuarzo sin choque, lo que bien puede corresponder a presiones de $14.5 \mathrm{GPa}$, de acuerdo al trabajo experimental de McMillan et al. (1992). Por lo tanto, esta presión podría inferirse debida al impacto además de la temperatura extrema. Un enfriamiento posterior formó cristobalita en la fulgurita Zacatecas (Figura 6), la cual es estable entre 1,470 y $1,625{ }^{\circ} \mathrm{C}$ (Goedeke y Techmer, 1999), y wollastonita (Figura 3b-3d, 3f) con temperatura de formación de alrededor de $900^{\circ} \mathrm{C}$ (Deer et al., 1978). Las vesículas posiblemente indican evaporación de agua y otros constituyentes en el momento de la fusión a temperaturas cercanas a $\operatorname{los} 2,000^{\circ} \mathrm{C}$ (Grapes, 2006).

La temperatura elevada generada por el relámpago, además del vidrio, formó compuestos como son las pequeñas partículas metálicas de composición variable de $\mathrm{Si}$-Fe (siliciuros), Fe-Si-Al y los compuestos con As-Ca, no reportados anteriormente en fulguritas. En la matriz vítrea o sobre la superficie de ésta (Figura 5) estos compuestos son poco comunes y se sabe que se forman en ambientes de reducción extrema (Essene y Fisher, 1986; Donaldson, 1976; Osinski, 2003). Según Wasserman et al. (2002) estos siliciuros son los productos finales de enfriamiento luego de la fusión a temperatura extremadamente alta, lo que se observa en la fulgurita de Zacatecas. Este tipo de materiales existe en forma más abundante sólo en la superficie de algunas costras vítreas, formando cristales granulares que pueden estar alineados (Figura 5d). En estas fulguritas los siliciuros son submicroscópicos; en otros lugares se han reportado de forma esférica y tamaños milimétricos y centimétricos, probablemente debido a intensidad y duración del fenómeno. Aleaciones de $\mathrm{Si}-\mathrm{Fe}-\mathrm{Al}$ de tamaños y composiciones similares a las de la fulgurita Zacatecas, también han sido estudiadas en la fulgurita de Bustaviejo (Madrid) por García-Guinea et al. (2009).

Otro factor que interviene en la formación de estos productos

Tabla 5. Análisis seleccionados de aleaciones granulares y prismáticas de Fe-Si-Al de la Fulgurita Zacatecas obtenidos por energía dispersiva de rayos X (EDS). Normalizados al $100 \%$.

\begin{tabular}{lccccc}
\hline \multicolumn{5}{c}{ Proporción de elementos (\% en peso) } \\
\hline $\mathrm{Si}$ & 4.52 & 4.77 & 5.42 & 19.89 & 49.78 \\
$\mathrm{Ti}$ & 0.82 & 0.80 & 0.34 & 0.75 & 1.30 \\
$\mathrm{Al}$ & 5.75 & 7.75 & 3.93 & 6.25 & 1.69 \\
$\mathrm{Fe}$ & 89.00 & 86.68 & 90.30 & 73.11 & 47.23 \\
Total & 100 & 100 & 100 & 100 & 100 \\
\hline
\end{tabular}




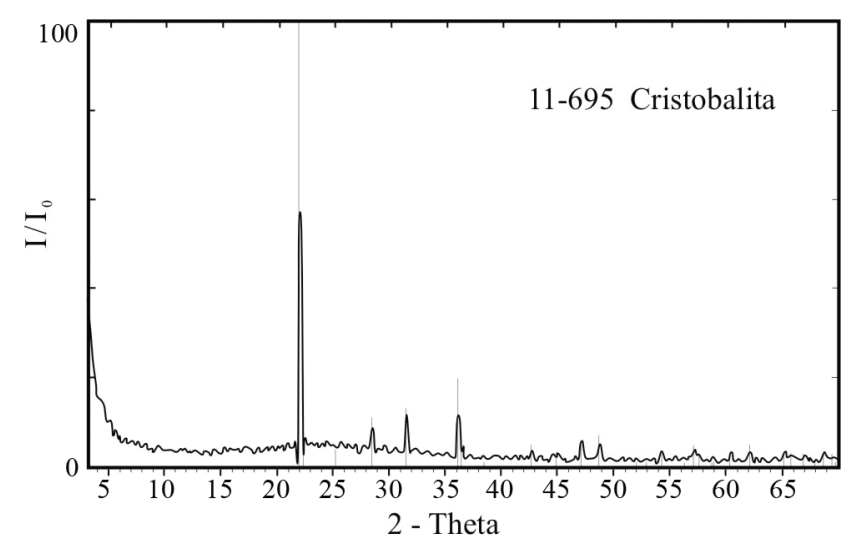

Figura 6. Espectro por difracción de rayos X, característico de la cristobalita, formada en la fulgurita Zacatecas.

reductores es la cantidad de materia orgánica contenida en el sustrato, puesto que en el proceso el carbón combina con el oxígeno en la fase de vapor dejando así el líquido silicatado reducido (Wasserman et al., 2002; Essene and Fisher, 1986). Es probable que en la fulgurita Zacatecas, formada en un suelo de cultivo, la cantidad de materia orgánica, el enriquecimiento en calcio y los fluídos del sustrato, intervinieron para que en esta fulgurita se formaran siliciuros, y compuestos de As-Ca y wollastonita. Los siliciuros, a pesar del tamaño tan pequeño que presentan, tienen formas cristalinas prismáticas indicando condiciones muy diferentes de formación con respecto a otros siliciuros de gran tamaño reportados en otras fulguritas y meteoritas. Este mecanismo de reducción extrema aún no es bien entendido (Essene y Fisher, 1986; Sheffer y Dyar, 2004; Ramírez-Cardona et al., 2006).

Por otra parte, al sureste del poblado de San José de Lourdes se encuentra el distrito minero de Fresnillo. De acuerdo a Mayorga- Ávila et al. (2011) los suelos de los alrededores se encuentran contaminados con arsénico y plomo. Por lo que el As presente en la fulgurita Zacatecas puede provenir de la contaminación de los alrededores (Tabla 4).

Por la gran cantidad de material fulgurítico y por las características mostradas, probablemente esta fulgurita se formó por la combinación de energías del choque del relámpago y de una fuente de alto voltaje, ya que se observó los restos de un poste de energía eléctrica que posiblemente fue destruido por el rayo y que a la fecha de la visita de campo todavía se encontraban a un lado del área de formación de las fulguritas. De ser así, se trataría más bien de una seudofulgurita o fulgurita híbrida o mixta con aporte natural para su formación por el relámpago. Podría

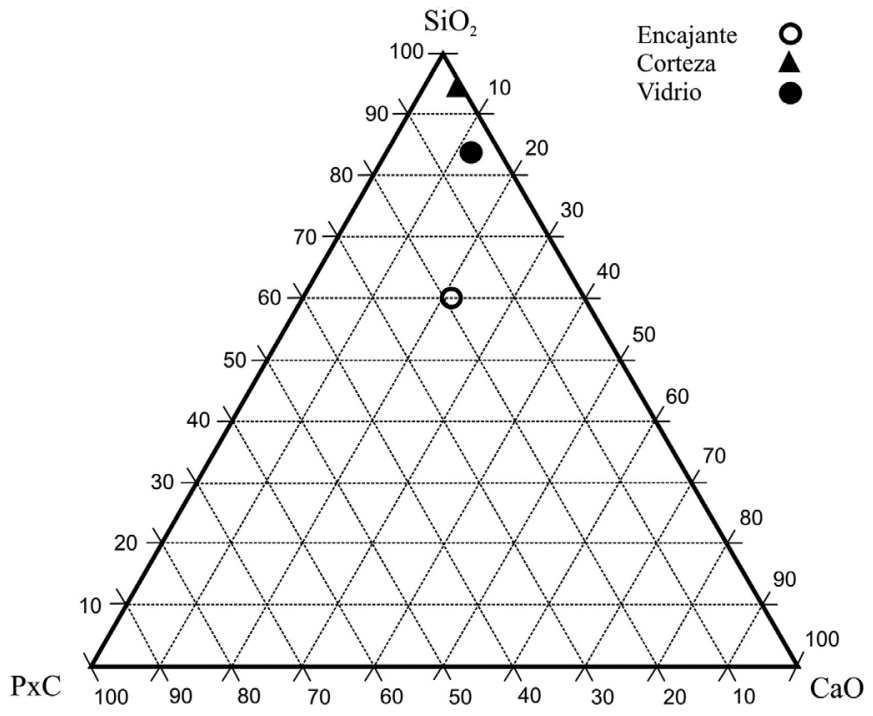

Figura 7. Resultados del análisis por fluorescencia de rayos $\mathrm{X}$ de la fulgurita Zacatecas y su material encajonante o madre. Contenido relativo de $\mathrm{SiO}_{2}-\mathrm{CaO}-$ PxC (pérdida por calcinación).

reservarse el término de seudofulgurita únicamente a las formadas experimentalmente sin intervención de un evento natural (Jones et al., 2005). Podría suponerse que los siliciuros se forman cuando han tenido intervención de energías de alto voltaje además del aporte del relámpago como la fulgurita Farmington de Connecticut (Sheffer et al., 2003, 2006) y la del Rosario, (Ramírez-Cardona et al., 2006); ambas presentan esferoides grandes de siliciuros a diferencia de la fulgurita Zacatecas con siliciuros micrométricos; posiblemente el tamaño de los siliciuros se deba a la duración e intensidad del evento debido al aporte externo de energía eléctrica. Sin embargo, otros factores no conocidos pudieron contribuir ya que la fulgurita descrita por Essene y Fisher (1986), con grandes esferoides de siliciuros, no menciona intervención alguna de una fuente energética aparte de la de los relámpagos.

\section{CONCLUSIONES}

Se describe y caracteriza una fulgurita en la localidad de Fresnillo, Zacatecas. Estas fulguritas se formaron a partir de suelos ricos en calcio, constituyendo vidrios heterogéneos ricos en calcio, de composición química similar a la de una andesita por su contenido en $\mathrm{SiO}_{2}$.

Tabla 6. Resultados del análisis de elementos mayores y traza por FRX de material encajonante (suelo), vidrio (fulgurita) y corteza (parte externa) de la fulgurita de Zacatecas.

\begin{tabular}{|c|c|c|c|c|c|c|c|c|c|c|c|c|c|c|}
\hline & $\underset{\%}{\mathrm{SiO}_{2}}$ & $\begin{array}{c}\mathrm{TiO}_{2} \\
\%\end{array}$ & $\begin{array}{c}\mathrm{Al}_{2} \mathbf{O}_{3} \\
\%\end{array}$ & $\begin{array}{c}\mathbf{F}_{2} \mathbf{O}_{3} \mathbf{t} \\
\%\end{array}$ & $\underset{\%}{\mathrm{MnO}}$ & $\underset{\%}{\mathbf{M g O}}$ & $\underset{\%}{\mathrm{CaO}}$ & $\begin{array}{c}\mathrm{Na}_{2} \mathrm{O} \\
\%\end{array}$ & $\begin{array}{c}\mathbf{K}_{2} \mathbf{O} \\
\%\end{array}$ & $\begin{array}{c}\mathbf{P}_{2} \mathbf{O}_{5} \\
\%\end{array}$ & \multicolumn{2}{|c|}{$\underset{\%}{\mathbf{P} \times \mathbf{C}}$} & \multicolumn{2}{|c|}{ Suma } \\
\hline Encajonante & 51.28 & 0.22 & 8.30 & 2.13 & 0.12 & 1.11 & 18.06 & 0.38 & 2.25 & 0.07 & 16.11 & 100.03 & \multicolumn{2}{|c|}{100.03} \\
\hline Vidrio & 60.84 & 0.28 & 10.13 & 2.47 & 0.04 & 1.33 & 18.56 & 0.47 & 2.80 & 0.07 & 3.21 & 100.20 & \multicolumn{2}{|c|}{100.20} \\
\hline & $\begin{array}{c}\mathbf{R b} \\
\mathrm{ppm}\end{array}$ & $\begin{array}{c}\mathrm{Sr} \\
\mathrm{ppm}\end{array}$ & $\begin{array}{c}\text { Ba } \\
\text { ppm }\end{array}$ & $\begin{array}{c}\mathbf{Y} \\
\mathrm{ppm}\end{array}$ & $\begin{array}{c}\mathbf{Z r} \\
\mathrm{ppm}\end{array}$ & $\begin{array}{c}\mathbf{N b} \\
\mathrm{ppm}\end{array}$ & $\begin{array}{c}\mathbf{V} \\
\mathrm{ppm}\end{array}$ & $\underset{\mathrm{ppm}}{\mathrm{Cr}}$ & $\begin{array}{c}\text { Co } \\
\text { ppm }\end{array}$ & $\begin{array}{c}\mathbf{N i} \\
\mathrm{ppm}\end{array}$ & $\begin{array}{c}\mathrm{Cu} \\
\mathrm{ppm}\end{array}$ & $\begin{array}{c}\text { Zn } \\
\text { ppm }\end{array}$ & $\begin{array}{c}\text { Th } \\
\text { ppm }\end{array}$ & $\begin{array}{c}\mathbf{P b} \\
\text { ppm }\end{array}$ \\
\hline Corteza & 145 & 378 & 512 & 45 & 175 & 18 & 57 & 20 & 24 & 14 & 18 & 54 & 18 & 13 \\
\hline Vidrio & 194 & 148 & 529 & 62 & 243 & 29 & 65 & 27 & 20 & 19 & 22 & 82 & 26 & 29 \\
\hline
\end{tabular}




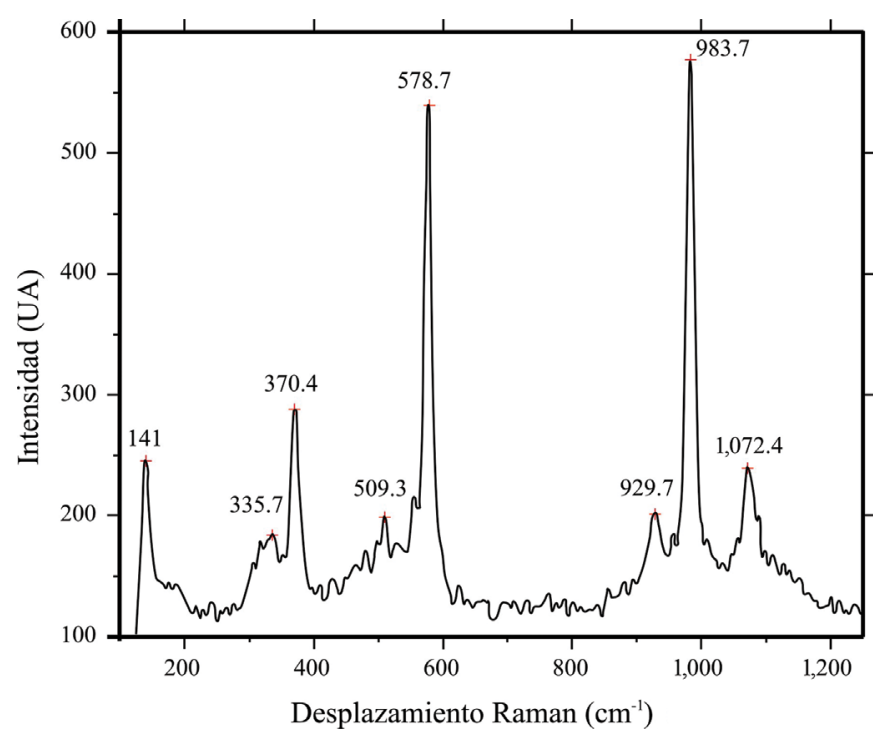

Figura 8. Espectro de micro-Raman mostrando los picos característicos de la wollastonita en la fulgurita Zacatecas.

Esta fulgurita se caracteriza por la presencia de algunas estructuras cristalinas: cristobalita, formas incipientes de wollastonita (reportados por primera vez en una fulgurita) y un silicato indiferenciado rico en Fe. Además, se encontraron aleaciones cristalinas micrométricas raras de siliciuros en distintas proporciones de $\mathrm{Si}-\mathrm{Fe}$, que no forman agregados esféricos, compuestos de $\mathrm{Si}$-Fe-Al y de As-Ca; estos últimos se reportan por primera vez en fulguritas. También se ridentificó una textura similar a la del cuarzo ballen, la cual es característica en algunas estructuras de impacto meteorítico.

La fulgurita Zacatecas claramente muestra materiales de extrema reducción con algunos de éstos similares a los encontrados en otras fulguritas. La reducción parece ser intrínseca a los procesos de alta temperatura de silicatos causados por relámpagos, en este caso estimada con un valor mínimo de $1700^{\circ} \mathrm{C}$. Debido a la posibilidad de aportación incidental de energía eléctrica por una fuente de alto voltaje, a la fulgurita aquí descrita podría llamársele seudofulgurita.

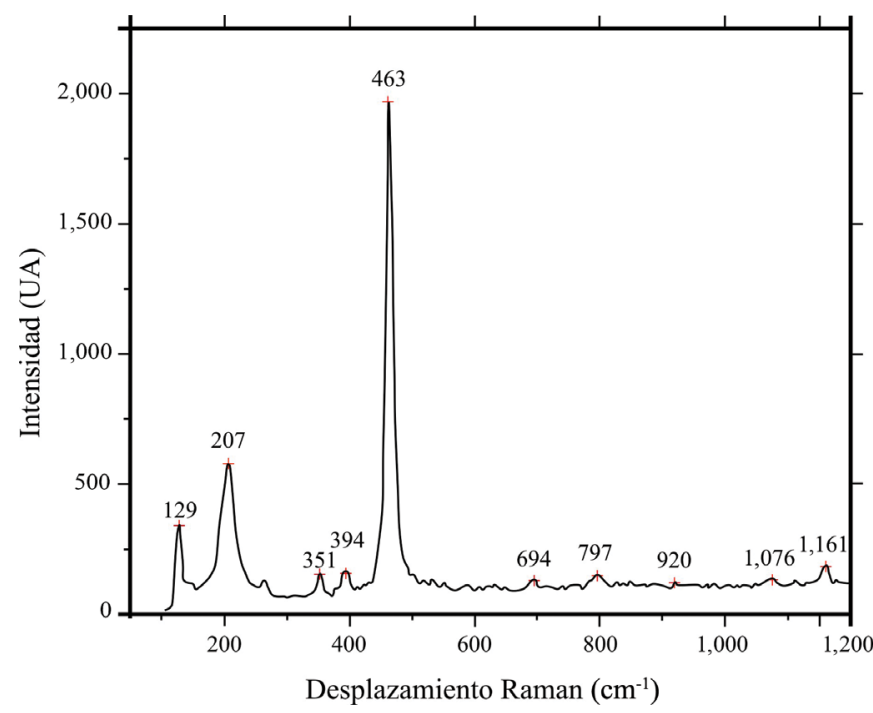

Figura 9. Espectro de micro-Raman mostrando los picos característicos de cuarzo (el máximo en $463 \mathrm{~cm}^{-1}$ ) en la fulgurita Zacatecas.

\section{AGRADECIMIENTOS}

Este trabajo se realizó gracias al apoyo financiero otorgado por CONACyT 43227. Agradecemos a los árbitros Dr. Francisco A. Paz Moreno, Dr. Eduardo González Partida, a otros anónimos y al editor Dr. Edgar R. Santoyo Gutiérrez por la revisión crítica y exhaustiva a este trabajo; a Sonia Ángeles y a Carlos Linares por su asistencia con el microscopio electrónico de barrido y a Diego Aparicio por la elaboración de las secciones delgadas.

\section{REFERENCIAS}

Bernal, J.P., Lozano-Santacruz, R., 2005, Characterization of a new set of eight geochemical reference materials for XRF major and trace element analysis: Revista Mexicana de Ciencias Geológicas, 22(3), 329-344.

Bird, J.M., Weathers, M.S., 1975, Josephinite: Specimens from the earth's core?: Earth and Planetary Science Letters, 28(1), 51-64.

Bryan, W.B., 1972, Morphology of quench crystals in submarine basalts: Journal of Geophysical Reasearch, 77(29), 5812-5819.

Cartens, H., 1975, Thermal history of impact melt rocks in the Fennoscandian shield: Contributions to Mineralogy and Petrology, 50, 145-155

Carter, E., Pasek, M.A., Smith, T., Kee, T.P., Hines, P., Howell, G., M., E., 2010, Rapid Raman mapping of a fulgurite: Analytical and Bioanalalytical Chemistry, 397, 2647-2658.

Deer, W.A., Howie R.A., Zussman J., 1978, Single-chain silicates, Rock Forming Minerals: New York, Longman Scientific, Second edition, 668 pp.

Donaldson, C.H., 1976, An experimental investigation of olivine morphology: Contributions to Mineralogy and Petrology, 57, 187-213.

Ege, C., 2005, What are fulgurites and where can they be found?: Utah Geological Survey, Survey Notes, 37, 1.

Ende, M., Schorr, S., Kloess, G., Franz, A., 2012, Shocked quartz in Sahara fulgurite: European Journal of Mineralogy, 24, 3, 499-507.

Essene, E.J., Fisher, D.C., 1986, Lightning strike fusion: extreme reduction and metal-silicate liquid inmiscibility: Science, 234, 189-193.

Ferriére, L., Koeberl, C., Reimold, W.U., 2009, Characterisation of ballen quartz and cristobalite in impact breccias: new observations and constraints on ballen formation: European Journal of Mineralogy, 21, 203-217.

García-Guinea, J., Furio, M., Fernández-Hernan, M., Bustillo, M.A., CrespoFeo, E., Correcher, L., Sánchez-Muñoz, L., Matezanz, E., 2009, The quartzofeldespathic fulgurite of Bustaviejo (Madrid): glassy matrix and Silicon phases en Proceedings of the Conference on Micro-Raman Spectroscopy and Luminescence Studies in the Earth and Planetary Sciences: Mainz, Germany, American Institute of Physics, 34-35.

Gifford, A.C., 1999, Clay soil fulgurites in the eastern goldfields of Western Australia: Journal of the Royal Society of Western Australia, 82, 165-168

Goedeke, D., Techmer, K., 1999, Partial melting in fulgurites from Little Ararat (Turkey) and Dissistock (Swiss Alps): European Union of Geoscience, 10, Journal of Conference Abtracts, 4(1).

Gopon, P., Fournelle, J., Sobol, P., Spicuzza, M., Pinar, P., Richter, S., Llovet, X., Valley J.W., 2014, Soft X-Ray EPMA Analyses of Extremely Reduced Phases from Apollo 16 Regolith: Problems and Solutions for Sub-Micron Analysis: Microscopy and Microanalysis, 20 (Suppl. 3), 698-699.

Grapes, R., 2006, Pyrometamorphism: Germany, Springer Freiburg, 275 pp.

Hawkins, G.S., 1960, Analysis of a supposed clay fulgurite from Ontario: Nature, 185, 301.

Heide, K., Heide, G., 2011, Vitreous state in nature-origin and properties: Chemie der Erde, 71, 305-335.

Hörz, F., Mittlefehldt, D.W., See T.H., Galindo, C., 2002, Petrographic studies of the impact melts from Meteor Crater: Meteoritics and Planetary Science, 37, 501-531.

INEGI (Instituto Nacional de Estadística, Geografía e Informática), 2007, Conjunto de Datos Vectorial Edafológico, Serie II, escala 1:2500000 (Contínuo Nacional). México. http://inegi.org.mx/map/

Jones, B.E., Jones, K.S., Rambo, K.J., Harkov, V.A., Jerald, J., Uman, M.A., 2005, Oxide reduction during triggered-lightning fulgurite formation: Journal of Atmospheric and Solar-Terrestrial Physics, 67, 423-428.

Kuriyama, N., Nakamura, J., Muto, J., Nagase, T., Pati, J.K., 2011, “Ballen quartz” 
from the Dhala impact structure and its crystal orientation pattern by electron back scattered diffraction (EBSD), en $42^{\text {nd }}$ Lunar and Planetary Science Conference: The Woodlands Texas, USA, Lunar and Planetary Institute, abstract 1657.

Lofgren, G., 1971, Spherulitic textures in glassy and crystalline rocks: Journal of Geophysical Reasearch, 76, 5635-5648.

Lozano-Fernández, R.P., González-Laguna, R., Martin-Crespo, T., 2007, Descripción macroscópica de la fulgurita de Torre de Moncorvo (Portugal): Geogaceta, 42, 139-142.

Lysiuk, A., Lysiuk, G.N., 2005, Spectroscopy of the fulgurite glasses: Geophysical Research Abstracts, 7, 00682.

Macdonald, F. A., Mitchell K., Cina, S.E., 2004, Evidence for a lightning-strike origin of the Edeowie glass, en XXXV Lunar and Planetary Science Conference: Texas, USA, abstract 1406.

Mayorga-Ávila, R.E., Carrizales-Yáñez, L., Díaz-Barriga Martínez, F., RazoSoto I., 2011, Concentraciones de plomo y arsénico en suelo superficial del distrito minero Fresnillo, Zacatecas, en XXI Congreso Nacional de Geoquímica: Monclova, Coahuila, México, Actas INAGEQ, 17(1), 166.

McMillan, P.F., Wolf G.H., Lambert P., 1992, A Raman spectroscopic study of shocked single crystalline quartz: Physics and Chemistry of Minerals, 19, 71-79.

Mokhtari, A., 2014, Evidence of shock metamorphism effects in suevite from the Triffa Plain in North Eastern Morocco: Universal Journal of Applied Science, 2(4), 77-82.

Ortíz-Hernández, L.E., Hernández-Pérez, I., 1996, Pseudofulgurita El Rosario: un agregado vítreo exótico en el estado de Hidalgo: Boletín de la Sociedad Geológica Mexicana, 52(3-4), 43-51.

Osinski, G.R., 2003, Impact glasses in fallout suevites from Ries impact structure, Germany: An analytical SEM study: Meteoritcs and Planetary Science, 38(11), 1641-1667.

Parnell, J., Thackrey, S., Muirhead,A., Wright, A., 2008, Transient hightemperature processing of silicates in fulgurites as analogues for meteorite and impact melts, en XXXIX Lunar and Planetary Science Conference: League City, Texas, USA, Lunar and Planetary Institute, abstract 1286.

Ramírez-Cardona, M., Flores-Castro, K., Cortés-García, P.P., Ortiz-Hernández, L.E., 2006, Mineralogical study of binary iron silicides (Fe-Si system) in a fulgurite from Hidalgo, México: Boletín de Mineralogía 17, 69-76.
Reyes-Salas A.M., Ortega-Gutiérrez, F., Macías-Romo, C., Girón P., Cervantes, K., García, J., Flores-Gutiérrez, D., Linares-López, C., 2006, Fulguritas de Zacatecas: un estudio petrográfico, geoquímico y mineralógico: Boletin de Mineralogía, 17, 67.

Riley, C.M., 1959, An iron fulgurite from Nebraska: Bulletin of The University of Nebraska State Museum, 4(5).

Servicio Geológico Mexicano, 1998, Carta geológico-minera Fresnillo F13-3, escala 1:250000: Pachuca, Hidalgo, Secretaría de Economía, 1ª. Edición.

Sheffer, A.A., Dyar, M.D., 2004, ${ }^{57} \mathrm{Fe}$ Mössbauer spectroscopy of fulgurites: Implications for chemical reduction, en XXXV Lunar and Planetary Science Conference: Texas, USA, Lunar and Planetary Institute, abstract 1372.

Sheffer, A.A., Melosh, H.J., Jarnot B.M., Lauretta D.S., 2003, Reduction of silicates at high temperature: fulgurites and thermodynamic modeling, en XXXIV Lunar and Planetary Science Conference: League City, Texas, USA, Lunar and Planetary Institute, abstract 1467.

Sheffer, A.A., Dyar, M.D., Sklute, E.C., 2006, Lightning strike glasses as an analog for impact glasses: ${ }^{57} \mathrm{Fe}$ mössbauer spectroscopy of fulgurites, en XXXVII Lunar and Planetary Science Conference: League City, Texas, USA, Lunar and Planetary Institute, abstract 2009.

Schmieder, M., Buchner, E., 2007, Is "ballen quartz" diagnostic for shock metamorphism?: Geochimica et Cosmochimica Acta, 71, A897.

Switzer, G., Melson, W.G., 1972, Origin and composition of rock fulgurite glass: The Earth Scientist, 9, 12 pp.

Wasserman, A.A., Melosh, H.J., 2001, Chemical reduction of impact processed materials en 32nd Annual Lunar and Planetary Science Conference: Houston, Texas, Lunar and Planetary Institute, abstract 2037.

Wasserman, A.A., Melosh, H.J., Lauretta, D.S., 2002, Fulgurites: a look at transient high temperature processe in silicates, en XXXIII Lunar and Planetary Science Conference: League City, Texas, USA, Lunar and Planetary Institute, abstract 1308.

Manuscrito recibido: marzo 6, 2017

Manuscrito corregido recibido: junio 5, 2017

Manuscrito aceptado: junio 6, 2017 


\section{Fe de erratas / Erratum}

Fe de erratas a Estudio petrográfico, geoquímico y mineralógico de la fulgurita San José de Lourdes, Zacatecas, México [Rev. Mex. Cienc. Geol., 34 (2017), 170-181]

\section{Adela Margarita Reyes-Salas ${ }^{1, *}$, Consuelo Macías-Romo ${ }^{1}$, Fernando Ortega-Gutiérrez ${ }^{1}$, Eugenio Octavio Reyes-Salas ${ }^{2}$, Daniel Flores-Gutiérrez ${ }^{3}$, Leticia Alba-Aldave', Patricia Girón-García ${ }^{1}$, Jasinto Robles-Camacho ${ }^{4}$, José Luis García-Martínez ${ }^{1}$ y Karina Elizabeth Cervantes-de la $\mathrm{Cruz}^{5}$}

${ }^{1}$ Universidad Nacional Autónoma de México, Instituto de Geología, Del. Coyoacán, 04510, Ciudad de México, México.

${ }^{2}$ Universidad Nacional Autónoma de México, Facultad de Química, Del. Coyoacán, 04510, Ciudad de México, México.

${ }^{3}$ Universidad Nacional Autónoma de México, Instituto de Astronomía, Del. Coyoacán, 04510, Ciudad de México, México.

${ }^{4}$ Instituto Nacional de Antropología e Historia, Subdirección de Laboratorios y Apoyo Académico, Centro INAH Michoacán, México.

${ }^{5}$ Universidad Nacional Autónoma de México, Facultad de Ciencias, Del. Coyoacán, 04510, Ciudad de México, México.

*adelars@unam.mx

\footnotetext{
En el artículo indicado arriba, dentro de la Figura 3 hay varias omisiones en cuanto a las descripciones y las escalas. A continuación se presenta la Figura 3 con la informacíon completa. Los editores lamentan el error.

In the above article, on the Figure 3 are several mistakes related to the descriptions and scales. The Figure 3 is presented in the next page with the complete information. The editors apologize for this mistake.
} 

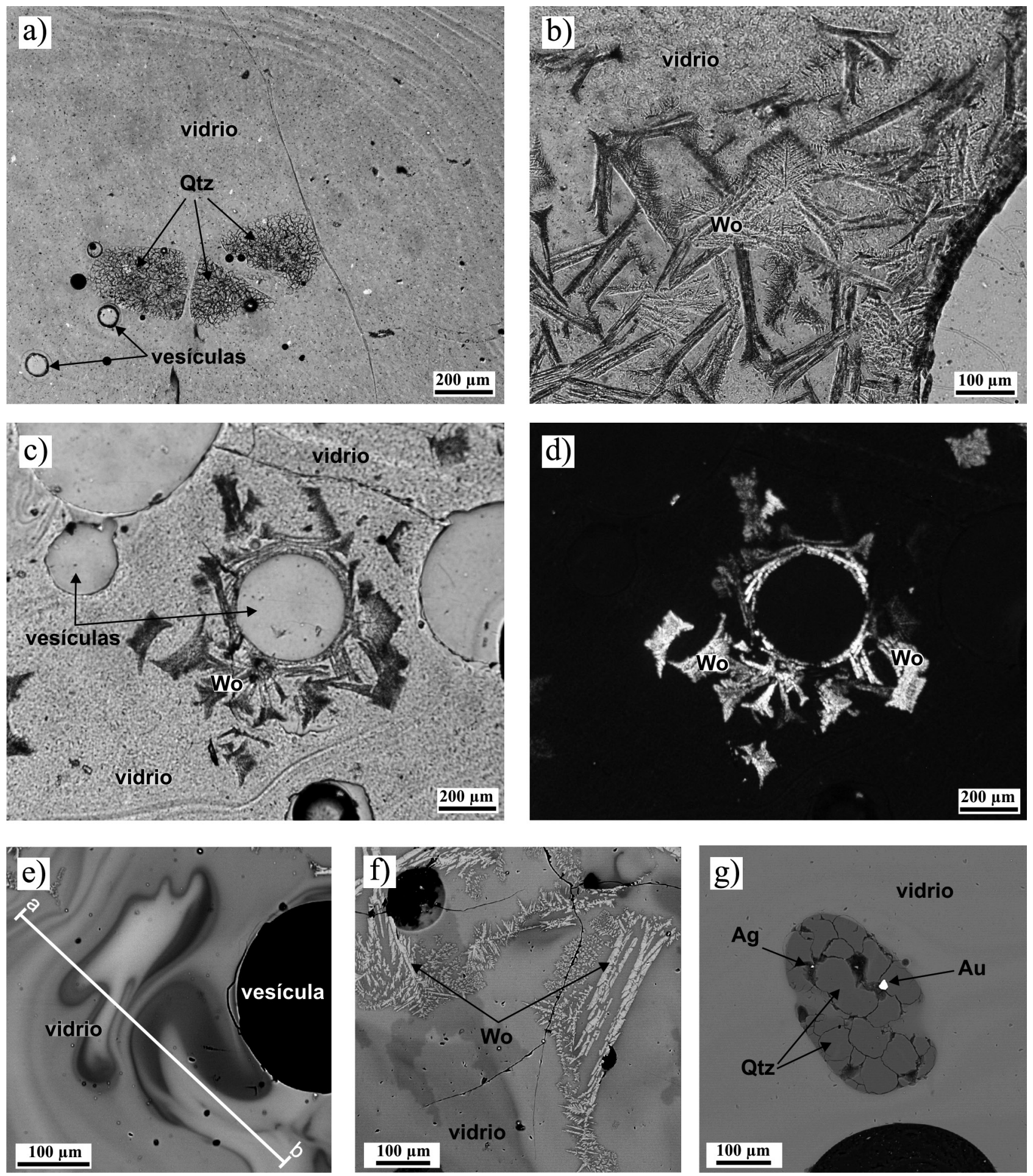

Figura 3. Fotomicrografías tomadas con microscopio petrográfico: a-c) Con luz natural; d) con luz polarizada. a) Parte del vidrio que conforma a la fulgurita Zacatecas con textura fluidal, pequeñas vesículas y hacia el centro, un esferoide alargado y separado en tres porciones formado por granos de $\mathrm{SiO}_{2}$. b) $\mathrm{Cristalitos}$ aciculares de wollastonita inmersos en vidrio, creciendo alrededor de una vesícula; al centro, textura plumosa. c) y d) Morfologías esqueléticas de wollastonita alrededor de una vesícula. e-g) Imágenes tomadas con electrones retrodispersados en el microscopio electrónico. e) Muestra, en tonos diferentes, la composición heterogénea del vidrio; los tonos más claros corresponden a los puntos analizados con mayor cantidad de aluminio y hierro. f) Cristales incipientes de wollastonita. g) Grano de cuarzo ballen el cual contiene dos partículas de oro y plata nativa; a la derecha, la partícula más brillante es oro. 